\title{
Chapter 8 \\ Insights from Computer Modeling: Analysis of Physical Characteristics of Glioblastoma in Patients Treated with Tumor-Treating Fields
}

\author{
Edwin Lok, Pyay San, and Eric T. Wong
}

\subsection{Introduction}

Tumor-treating fields (TTFields) are intermediate frequency electric fields at $200 \mathrm{kHz}\left(\mathrm{kHz}\right.$ or $\left.10^{3} \mathrm{~Hz}\right)$ that have anti-tumor activity. In a pivotal phase III clinical trial for newly diagnosed glioblastoma patients, the addition of TTFields to maintenance temozolomide, which was administered after initial radiotherapy and concurrent daily temozolomide, was found to improve both progression-free survival and overall survival when compared to those who only received maintenance temozolomide $[1,2]$. This positive trial result led the US FDA to approve the use of TTFields for these patients and the incorporation of this therapy into the National Comprehensive Cancer Network (NCCN) guidelines for malignant gliomas [3].

\subsection{TTFields Is Another Treatment Modality from the Electromagnetic Spectrum}

Energies from different parts of the electromagnetic spectrum are utilized to treat various types of malignancies. For glioblastoma, ionizing radiation from external beam radiotherapy is the mainstay of treatment at initial diagnosis. The frequency is in the ectahertz $\left(\mathrm{EHz}\right.$ or $\left.10^{18} \mathrm{~Hz}\right)$ range and the high energy results in direct DNA damage, such as double-strand DNA breaks; indirect effects are also a result, including the generation of oxygen radicals causing secondary tissue damage [4]. Major advances of the past decades consist of improving the conformality of the beam, as

E. Lok $\cdot$ P. San $\cdot$ E. T. Wong $(\bowtie)$

Brain Tumor Center \& Neuro-Oncology Unit, Department of Neurology, Beth Israel

Deaconess Medical Center, Harvard Medical School, Boston, MA, USA

e-mail: ewong@bidmc.harvard.edu 
in intensity-modulated radiation therapy and stereotactic radiosurgery, resulting in less radiation scatter to the surrounding tissue adjacent to the tumor $[5,6]$.

Laser interstitial thermal therapy (LITT) is another treatment for brain tumors that utilizes a specific part of the electromagnetic spectrum at the microwave frequency or $100 \mathrm{MHz}$ range. A probe is stereotactically inserted into the tumor target under MRI guidance, and the laser at the tip of the probe emits microwave energy to heat the tumor tissue while the temperature is being monitored [7]. The primary application is for the treatment of brain metastasis and radiation necrosis, but surgically inaccessible glioblastomas can also be treated.

TTFields therapy operates at a frequency of $200 \mathrm{kHz}$ for glioblastoma, which is based on preclinical data on mitotic interference of glioma cells in tissue culture [8]. This frequency is below radio frequency for the transmission of AM signals. There are a number of biological effects that can be exploited to treat malignancies. These include disruption of tubulin and septin, both of which are intracellular macromolecules possessing large dipole moments and are necessary for the orderly progression of mitosis in dividing cells [8,9]. Tubulin monomers coalesce to form microtubules and the mitotic spindle during metaphase and anaphase. These higher order structures are needed to align the 23 pairs of chromosomes in the mitotic plate and to guide the subsequent migration of the corresponding sister chromatids to the respective centrioles. Septin heterotrimers are needed for cytokinesis, or the contraction of the plasma membrane along the equatorial plane of the dividing cell, that eventually produces two daughter cells. Notable phenomena of violent membrane blebbing and asymmetric chromosome segregation have been observed under the influence of TTFields, resulting in aneupoloidy, mitotic arrest, and/or cellular stress that may trigger an immunogenic response [8-10].

\subsection{Quantifying Electric Field Delivery in the Brain}

The amount of electric field delivered to the tumor target can be quantified. However, the brain has a complex geometry consisting of multiple layers of folded tissues (such as dura, gray matter, white matter, and subcortical nuclei) and asymmetric spaces (ventricles and the subarachnoid space on the cerebral convexity). In addition, the glioblastoma is situated within the white matter with extension to the adjacent gray matter and displacement of the subarachnoid or ventricular space. This tumor is usually seen as an enhancing lesion on MRI, and its gross tumor volume (GTV) can be contoured and delineated by appropriate software. When solving for the electric field distribution at the GTV, the solutions to the differential equations are not straightforward and only representative numerical approximations can be computed using techniques such as finite element analysis. The accuracy of the solution depends on several factors: the smoothness of interface between structures and the resolution of the finite elements that form the approximate solid geometry of the tissue. There is always a trade-off between accuracy of the model and the computational requirements needed to generate a solution. In general, a larger 
number of elements that are used to represent a more accurate geometry will require more time and increased computational capability to generate a solution.

The workflow to generate a prediction for the numerical value of the electric field at the GTV requires a number of steps [11]. It begins with the conversion of the Digital Imaging and Communications in Medicine (DICOM) dataset from MRI into Neuroimaging Informatics Technology Initiative (NIfTI) image format using the conversion function from Statistical Parametric Mapping 8 (SPM8) and then coregistering to a template in Montreal Neurological Institute space. The default workflow generates ten binarized masks, including white matter, gray matter, cerebellum/brainstem, cerebrospinal fluid, orbits, skull, scalp, gel, electrodes, and air; it has been customized for the finite element analysis of TTFields. The binary masks are then imported into a 3D image processing software such as ScanIP (Synopsis, Mountain View, CA) for post-processing of generated errors. The boundary conditions are then specified and the presence of island cavities and artifacts are manually corrected to ensure no overlapping or missing boundaries are present. The Dice coefficient can then be used to measure the degree of overlap before and after manual processing. The optimized masks are then imported into a simulation software such as COMSOL Multiphysics (Stockholm, Sweden). The output of this process consists of visual maps of the distributed electric fields and the specific absorption rate (SAR) within the brain and the GTV.

The electric field-volume histogram $(\mathrm{EVH})$ and specific absorption rate-volume histogram (SARVH) are indispensable for the comparison of the electric fields and the SAR at the GTV between different models [12] (Fig. 8.1). The histograms are also applicable to other intracranial structures for monitoring side effects at regions adjacent to the GTV. Both EVH and SARVH also facilitate the comparison of TTFields in individual patients over time or between patients who are receiving the same treatment. A number of standardized parameters can be used, including $\mathrm{E}_{\mathrm{AUC}}$, $\mathrm{VE}_{150}, \mathrm{E}_{95 \%}, \mathrm{E}_{50 \%}$, and $\mathrm{E}_{20 \%}$ for electric field quantification, as well as $\mathrm{SAR}_{\mathrm{AUC}}$, $\mathrm{VSAR}_{7.5}, \mathrm{SAR}_{95 \%}, \mathrm{SAR}_{50 \%}$, and $\mathrm{SAR}_{20 \%}$ for specific absorption rate representation. In our prior modeling work, the scalp is the site having the highest overall electric field intensity and SAR because the transducer arrays are adjacent to the scalp. However, within the brain, the distribution is highly variable, with the frontal horns of the lateral ventricles and the genu of the corpus callosum having a higher electric field intensity and SAR due to their geometries and juxtaposition to cerebrospinal fluid $[12,13]$.

A number of factors can alter the intracranial distribution of TTFields. First, the thickness of the cerebrospinal fluid layer located in the subarachnoid space on the surface of the brain can change the penetration of TTFields into the GTV, which is located deeper within the white matter of the brain. In one of our models, a contraction of the cerebrospinal fluid layer by $1.0 \mathrm{~mm}$ increased the $\mathrm{E}_{\mathrm{AUC}}$ by $15-20 \%$, while an expansion by $0.5 \mathrm{~mm}$ can reduce the $\mathrm{E}_{\mathrm{AUC}}$ by about $10 \%$ [12]. Second, changes in the conductivity of GTV affect the distribution of TTFields inside the brain, but variations in permittivity do not. In particular, the presence of a tumor-associated necrotic core, which contains fluid and probably has a higher conductivity, may attenuate the penetration of TTFields into the tumor, likely by diverting the electric 

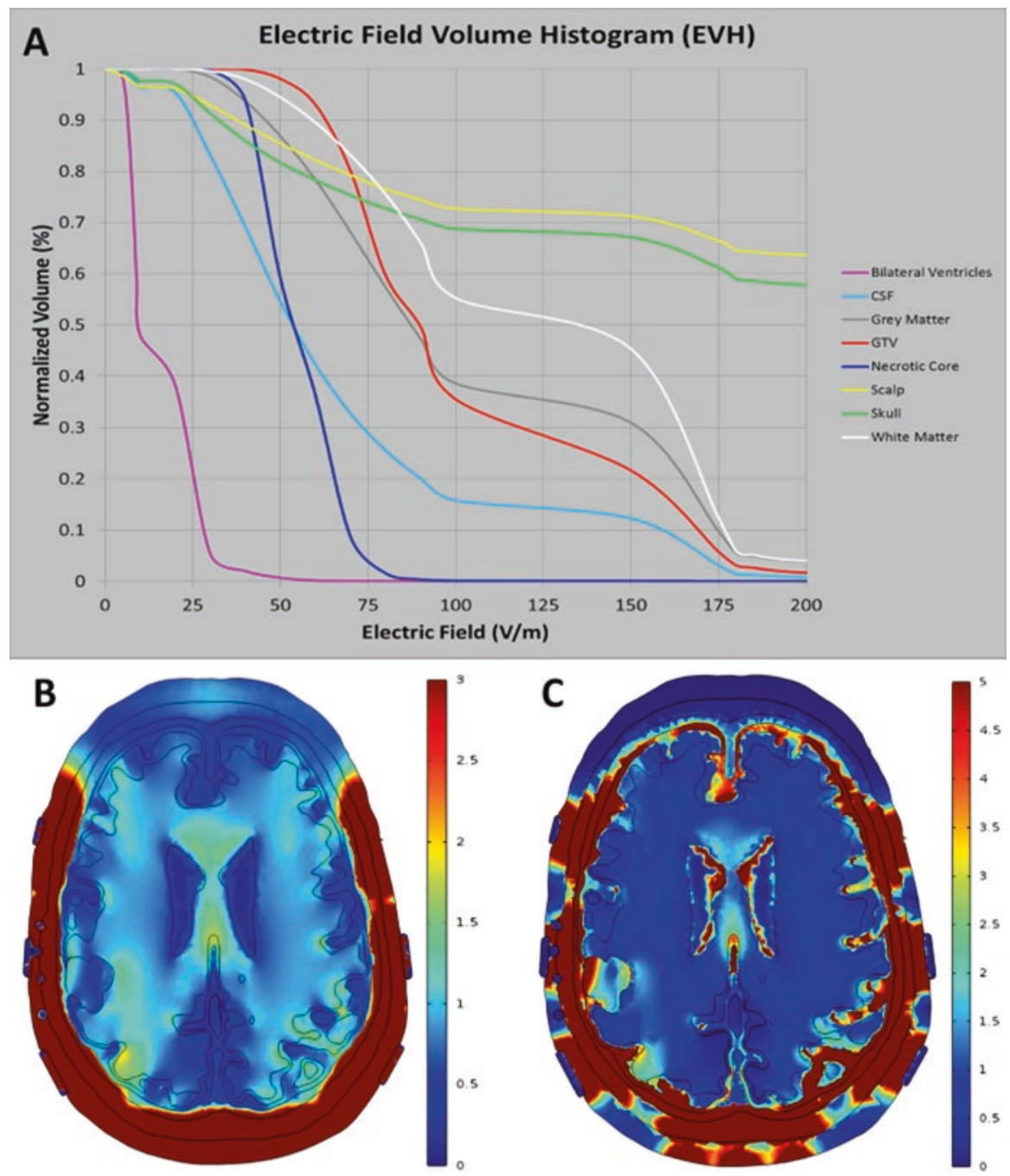

Fig. 8.1 (a) EVH for various tissue structures and GTV. Simulation results for (b) electric field and (c) SAR distributions on an axial plane within the brain, showing higher intensity near the GTV and the corpus callosum 
fields away from the active regions of the tumor. Therefore, these anatomic variations may alter the response of patients to TTFields treatments.

\subsection{Clinical Outcome from TTFields Treatment}

The efficacy of TTFields in the glioblastoma population has been tested in randomized phase III clinical trials $[1,2,14]$. The EF-11 trial was conducted in the recurrent glioblastoma population and patients were randomized in a 1:1 fashion to either TTFields monotherapy or best physician's choice chemotherapy [14]. Both progression-free survival and overall survival were comparable in the two cohorts, suggesting that TTFields is an equivalent therapy, but without the toxicities associated with the chemotherapies used in the recurrent setting. There is a unique side effect of scalp irritation caused by placement of the transducer arrays onto the patient's head and the energy deposition on the scalp, but this can be readily treated with corticosteroid ointment with minimal sequela.

The post hoc analyses of EF-11 yielded a number of important findings. First, the Patient Registry Dataset (PRiDe) contains treatment and health information data from patients who underwent TTFields therapy in real-world, clinical practice settings in the USA from 2011 to 2013 after the initial United States FDA approval. PRiDe revealed that patients lived longer when their TTFields usage compliance averages $75 \%$ or greater per day [15]. Furthermore, dexamethasone is an antiinflammatory drug commonly used in the glioblastoma population to counteract cerebral edema caused by the tumor. A separate post hoc analysis showed that patients who used a higher dose of $\geq 4.1 \mathrm{mg} /$ day had a shortened survival compared to those who used $<4.1 \mathrm{mg} /$ day [16]. Therefore, TTFields therapy in this population requires maximizing treatment compliance while minimizing dexamethasone usage in order to facilitate its anti-glioblastoma effect.

The EF-14 trial is another randomized phase III trial of newly diagnosed glioblastoma patients [2]. All eligible patients received initial radiation and daily temozolomide and were then randomized prior to entry into the post-radiotherapy adjuvant phase of temozolomide treatment. Patients were randomized to receive TTFields plus temozolomide or temozolomide alone, and the TTFields cohort had longer progression-free survival and overall survival compared to the control. Mild to moderate scalp toxicity underneath the transducer arrays was seen in $52 \%$ of patients who received TTFields and temozolomide compared to none in the control cohort. Systemic toxicities and health-related quality-of-life measures were comparable between the two groups $[2,17]$. 


\subsection{Conclusion}

TTFields therapy has established anti-cancer efficacy and the utilization of this treatment for glioblastoma has resulted in prolongation of patient survival. However, the distribution of TTFields within the brain is still shrouded in mystery. Finite element analysis provides a means for the numerical approximation of the distribution of TTFields within the brain and particularly within the GTV. Furthermore, EVH and SARVH permit the quantification of changes in electric fields and specific absorption rate over time, as well as the comparison of these parameters between individuals. Ultimately, the goal is to develop personalized TTFields treatment for each glioblastoma patient.

Acknowledgments This research was supported in part by A Reason To Ride research fund.

\section{References}

1. Stupp, R., Tallibert, S., Kanner, A. A., et al. (2015). Maintenance therapy with tumor-treating fields plus temozolomide vs temozolomide alone for glioblastoma: A randomized clinical trial. JAMA, 314, 2535-2543.

2. Stupp, R., Tallibert, S., Kanner, A. A., et al. (2017). Effect of tumor-treating fields plus maintenance temozolomide vs maintenance temzolomide alsone on survival in patients with glioblastoma: A randomized clinical trial. JAMA, 318, 2306-2316.

3. https://www.nccn.org/professionals/physician_gls/pdf/cns.pdf

4. Baskar, R., Dai, J., Wenlong, N., et al. (2014). Biological response of cancer cells to radiation treatment. Frontiers in Molecular Biosciences, 1, 24.

5. Bortfeld, T. (2006). IMRT: A review and preview. Physics in Medicine and Biology, 51, R363-R379.

6. Taylor, M. L., Kron, T., \& Franich, R. D. (2011). A contemporary review of stereotactic radiotherapy: Inherent dosimetric complexities and the potential for detriment. Acta Oncologica, 50, 483-508.

7. Sharma, M., Balasubramanian, S., Silva, D., et al. (2016). Laser interstitial thermal therapy in the management of brain metastasis and radiation necrosis after radiosurgery: An overview. Expert Review of Neurotherapeutics, 16(2), 223-232.

8. Kirson, E.D., Gurvich, Z., Schneiderman, R., et al. (2004). Disruption of cancer cell replication by alternating electric fields. Cancer Research, 64(9), 3288-3295.

9. Gera, N., Yang, A., Holtzman, T. S., et al. (2015). Tumor treating fields perturb the localization of septins and cause aberrant mitotic exit. PLoS One, 10, e0125269.

10. Kirson, E. D., Dbalý, V., Tovaryš, F., et al. (2007). Alternating electric fields arrest cell proliferation in animal tumor models and human brain tumors. Proceedings of the National Academy of Sciences, 104(24), 10152-10157.

11. Timmons, J. J., Lok, E., San, P., et al. (2017). End-to-end workflow for finite element analysis of tumor treating fields in glioblastomas. Physics in Medicine and Biology, 62, 8264-8282.

12. Lok, E., San, P., Hua, V., et al. (2017). Analysis of physical characteristics of tumor treating fields for human glioblastoma. Cancer Medicine, 6, 1286-1300.

13. Lok, E., Hua, V., \& Wong, E. T. (2015). Computed modeling of alternating electric fields therapy for recurrent glioblastoma. Cancer Medicine, 4, 1697-1699. 
14. Stupp, R., Wong, E. T., Kanner, A. A., et al. (2012). NovoTTF-100A versus physician's choice chemotherapy in recurrent glioblastoma: A randomized phase III trial of a novel treatment modality. European Journal of Cancer, 48, 2192-2202.

15. Mrugala, M. M., Engelhard, H. H., Tran, D. D., et al. (2014). Clinical practice experience with NovoTTF-100A ${ }^{\mathrm{TM}}$ system for glioblastoma: The patient registry dataset (PRiDe). Seminars in Oncology, 42, S4-S13.

16. Wong, E. T., Lok, E., Gautam, S., \& Swanson, K. D. (2015). Dexamethasone exerts profound immunologic interference on treatment efficacy for recurrent glioblastoma. British Journal of Cancer, 113, 232-241.

17. Taphoorn, M. J. B., Dirven, L., Kanner, A. A., et al. (2018). Influence of treatment with tumortreating fields on health-related quality of life of patients with newly diagnosed glioblastoma: A secondary analysis of a randomized clinical trial. JAMA Oncology, 4, 495-504.

Open Access This chapter is licensed under the terms of the Creative Commons Attribution 4.0 International License (http://creativecommons.org/licenses/by/4.0/), which permits use, sharing, adaptation, distribution and reproduction in any medium or format, as long as you give appropriate credit to the original author(s) and the source, provide a link to the Creative Commons license and indicate if changes were made.

The images or other third party material in this chapter are included in the chapter's Creative Commons license, unless indicated otherwise in a credit line to the material. If material is not included in the chapter's Creative Commons license and your intended use is not permitted by statutory regulation or exceeds the permitted use, you will need to obtain permission directly from the copyright holder.

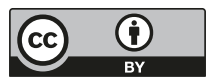

